\title{
Rare Sarcomatoid Carcinoma of the Liver in a patient with no history of Hepatocellular Carcinoma: A Case Report
}

\author{
Kevat B. Patel, MD', Rita H. Hawarny, MD'1, Elizabeth Saunders, MD', \\ Marc Hettlinger, MD ${ }^{1}$, Niru Nahar, MD'
}

ABSTRACT

Sarcomatoid carcinoma is a rare malignant tumor of unknown pathogenesis characterized by poorly differentiated carcinoma tissue containing sarcoma-like differentiation of either spindle or giant cell and rarely occurs in the gastrointestinal tract and hepatobiliary-pancreatic system. ${ }^{1}$ Primary hepatic sarcomatoid carcinoma accounts for only $0.2 \%$ of primary malignant liver tumors, and $1.8 \%$ of all surgically resected hepatocellular carcinomas. ${ }^{2}$ The majority of hepatic sarcomatoid carcinoma cases appear to occur simultaneously with hepatocellular or cholangiocellular carcinoma. ${ }^{3}$ The preferred treatment for hepatic sarcomatoid carcinoma is surgical resection and the overall prognosis is poor. ${ }^{4}$ This case depicts a 62-year-old female who underwent initial resection of a cavernous hemangioma in 2010. Seven years after her initial diagnosis, she developed what was initially felt to be local recurrence of the hemangioma but additional diagnostic workup with a liver biopsy confirmed primary hepatic sarcomatoid carcinoma.
Author affiliations are listed at the end of this article.

Correspondence to: Kevat Patel, MD Marshall University Joan C. Edwards School of Medicine patelke@marshall.edu

\section{KEYWORDS}

Sarcomatoid Carcinoma, Hepatocellular Carcinoma, Primary Hepatic Sarcomatoid Carcinoma

A 62-year-old female with past medical history significant for vegan diet, hypothyroidism, iron deficiency anemia, and cavernous liver hemangioma presented with weight loss and abdominal fullness for approximately one month with two days of acute altered mental status, fatigue, and weakness.

Patient had a complicated gastrointestinal history and underwent surgical resection of a cavernous hemangioma in 2010. Six years later, she developed abdominal fullness with right upper quadrant pain and an abdominal ultrasound at that time suggested hemangioma recurrence. In 2017, she underwent laparoscopy with unroofing of the hemangioma, drainage of an old organizing hematoma, removal of debris, and placement of an omental patch. Patient developed subsequent abdominal ventral hernia but a scheduled repair in January of 2018 was deferred due new onset of iron deficiency anemia with a hemoglobin of $6.7 \mathrm{~g} / \mathrm{dL}$. She underwent EGD and colonoscopy which revealed candida esophagitis and diverticulosis but no identifiable bleeding source. Her acute anemia was attributed to a nutritional deficit from her vegan diet so ferrous sulfate $325 \mathrm{mg}$ tablets three times a day were started. Abdominal CT scan showed a $13.6 \mathrm{~cm}$ mass adjacent to the gallbladder fossa, but further workup was deferred due to her anemia. In February, the patient's anemia persisted despite oral iron replacement therapy and she received infusion of feraheme $510 \mathrm{mg}$. She underwent subsequent EGD for reevaluation of anemia and repair of periumbilical hernia during the same month. In March, she was admitted for abnormal PET scan concerning for liver malignancy with maximum SUV of $20.1 \mathrm{~g} / \mathrm{mL}$. Liver abscess was diagnosed and CT guided drainage was performed. She was treated with amoxicillin/clavulanate and discharged home.

Three weeks later, she returned with abdominal 
pain, fatigue, poor appetite, intermittent fevers, altered mental status, and diarrhea. On examination, patient appeared uncomfortable, pale, and weak. Her medications included armour thyroid, ranitidine, and amoxicillin/clavulanate. Vitals included: pulse rate 127 , blood pressure $108 / 56$, temperature 101.8 , respiratory rate 20 and oxygen saturations of $98 \%$ on room air. Patient appeared drowsy but cooperative and not in distress. HEENT: normocephalic, pupils equal, round and reactive to light, extraocular movement intact. Respiratory: tachypneic with nonlabored respirations and decreased breath sounds bilaterally. Cardiovacular: tachycardic with normal rhythm. Gastrointestinal: soft, non-tender, nondistended, normal bowel sounds. Surgical incision sites were healing. Skin: warm, dry, pink with +2 pitting bilateral lower extremity edema.

Diagnostic data demonstrated leukocytosis of $15 \mathrm{~g} /$ $\mathrm{dL}$, hemoglobin of $6.0 \mathrm{~g} / \mathrm{dL}$, albumin of $1.5 \mathrm{~g} / \mathrm{dL}$, and normal liver enzymes with mildly elevated bilirubin level of $1.2 \mathrm{mg} / \mathrm{dL}$. CT scan of abdomen showed large heterogeneous lobular mass measuring $14.6 \mathrm{~cm} \mathrm{x}$ $10.8 \mathrm{~cm}$ in the superior portion of the right liver lobe with an inferior calcified cystic mass measuring $6 .{ }^{5}$ $\mathrm{cm} \times 5.3 \mathrm{~cm}$. Acute hepatitis panel, serum alpha-feto protein, carcinomembryonic antigen, and CA-19-9 were negative. Patient started on vancomycin and piperacillin/tazobactam, and transfused two units of PRBCs. Gastrointestinal PCR panel was positive for enteropathic Escherichia coli and Clostridium difficile so oral vancomycin was initiated. Additional anemia workup including bone marrow biopsy, EGD, and tagged RBC scan did not reveal source of anemia. CT enterography (Figure 1) showed large liver mass with liquefaction and air in inferior portion and a small tract of fluid between the mass and duodenal bulb concerning for fistulous tract.

Diagnosis of hepatic sarcomatoid carcinoma was obtained by liver biopsy with gross specimen demonstrating spindle shaped cells (Figures 2-4). ${ }^{1,5,6,7}$ Immunohistochemical panel additionally confirmed sarcomatoid carcinoma given expression of multiple cytokeratin (CAM 5.2, cytokeratin AE1/ AE3, oscar cytokeratin) and lack of reactivity for other lineage specific markers (Melan-A and SOX10, SMA, ALK, ERG, Desmin, CD21, CD3, CD20, CD15, CD30, PAX5, CD45, CD5, and S100). Sarcomatoid hepatocellular carcinoma and cholangiocarcinoma with sarcomatoid features were excluded as immunohistochemistry testing was negative for Hep Par-1, Arginase, Albumin ISH. The patient was transferred to a liver center and ultimately pursued

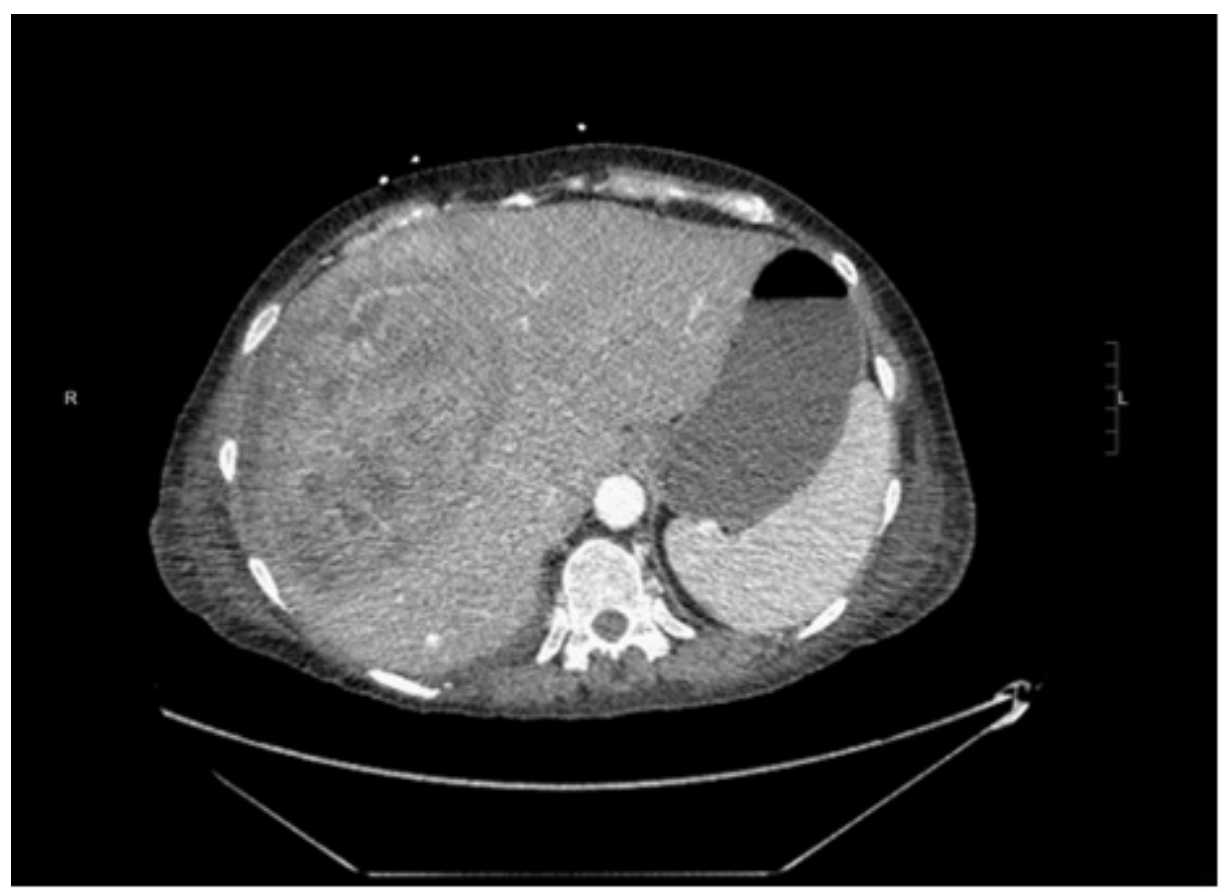

FIGURE 1: Large liver mass 


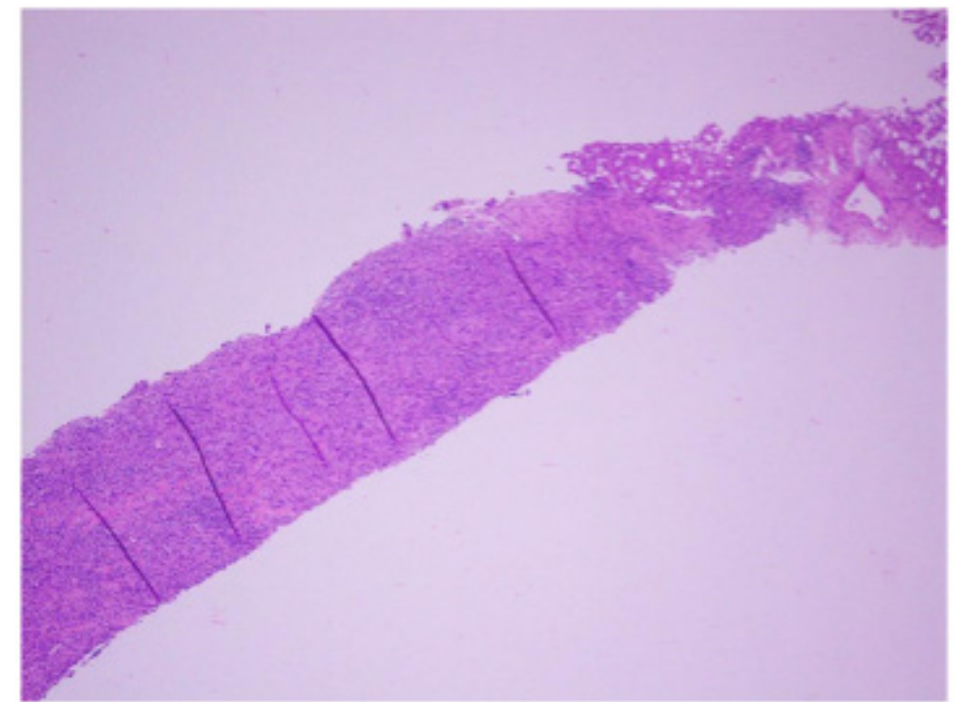

FIGURE 2: Low-power view predominantly showing a diffuse area of tumor with an area of benign liver with steatosis on the right side corner
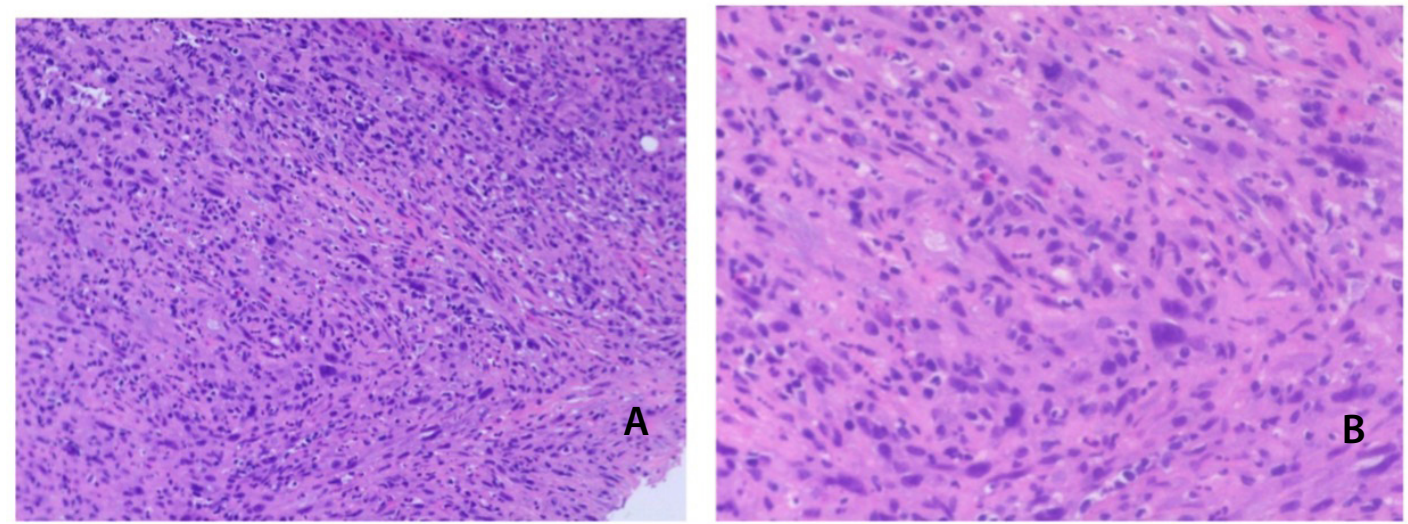

FIGURE 3: A) Low-power view showing spindle growth pattern of tumor, B) Highpower view showing spindle neoplastic cells with large cells
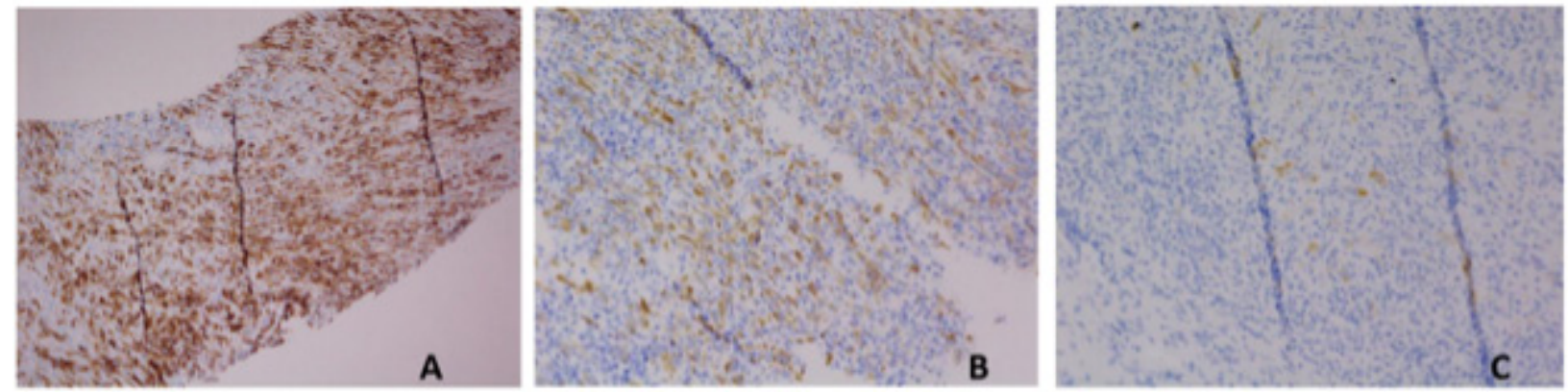

FIGURE 4: Immunohistochemical stains for multiple cytokeratins are positive for tumor - A) Oscar cytokeratin, B) CAMP5.2, and C) Pancytokeratin (AE1/AE3). 
hospice due to poor prognosis and passed away a few weeks later.

\section{DISCUSSION}

Hepatic sarcomatoid carcinoma is a rare malignant neoplasm of unclear pathogenesis. Presenting symptoms of sarcomatoid carcinoma are similar to that of hepatoceullar carcinoma with abdominal pain, weight loss, anorexia, and fatigue, which was the case for our patient. Hepatic sarcomatoid has a high risk of relapse, venous and intrahepatic invasion, and distant and lymph node metastasis at time of diagnosis. The preferred treatment for hepatic sarcomatoid carcinoma is surgical resection and the overall prognosis is poor. ${ }^{8}$

The present study reports a rare case of hepatic sarcomatoid carcionoma in a patient with a previous cavernous hemangioma. Previous studies have reported the usefulness of chemotherapy and literature review found one study with documentation of complete resolution of hepatic sarcomatoid carcinoma after seven cycles of doxorubicin and ifosfamide but no large-sample studies exist. ${ }^{9}$ Efficacy of alternative treatments such as radiotherapy, chemotherapy, and targeted therapy are unclear.

Liver conditions that have been associated with the development of liver sarcomatoid carcinoma include hepatitis $C$ virus (HCV) and hepatocellular carcinoma (HCC). ${ }^{10}$ Though liver malignancy can also be secondary to a primary GI sarcoma it is a rare or unknown cause of liver sarcomatoid carcinoma. In this particular case, the patient did not have a history of HCV, HCC or gastrointestinal malignancy. Her presentation was confounded by a history of iron deficiency anemia, and development of a hepatic abscess after surgical intervention to un-roof a recurring cyst which turned out to be hematoma. Cystic lesions of the liver are a common occurrence but rarely lead to primary liver neoplasms. The diagnosis of hepatic sarcomatoid remains elusive due its vague symptomatology and rarity. Additional studies are needed to explore possible adjunctive therapies to surgical resection to improve survival rates.

\section{AUTHOR AFFILIATIONS}

1. Marshall University Joan C. Edwards School of Medicine, Huntington, West Virginia

\section{REFERENCES}

1. Shinoda $M$, Shimazu M, Mukai M, et al. Spindle cell carcinoma of the intrahepatic bile duct in a patient with primary sclerosing cholangitis. Journal of Gastroenterology. 2003;38(11):10911096.

2. Leng Q, Xiang X, Tang Y, Yang Y, Qiu L. Primary hepatic sarcomatoid carcinoma: A case report. Experimental and Therapeutic Medicine. 2015;10(3):1145-1148.

3. Wang Q-B, Cui B-K, Weng J-M, Wu Q-L, Qiu J-L, Lin X-J. Clinicopathological characteristics and outcome of primary sarcomatoid carcinoma and carcinosarcoma of the liver. Journal of Gastrointestinal Surgery. 2012;16(9):1715-1726.

4. Giunchi F, Vasuri F, Baldin P, Rosini F, Corti B, D'Errico-Grigioni A. Primary liver sarcomatous carcinoma: report of two cases and review of the literature. Pathology - Research and Practice. 2013;209(4):249-254.

5. Nirei K, Matsuoka S, Moriyama M, Nakamura $\mathrm{H}$, Maebayashi T, Takayama T, Sugitani. M. Rare sarcomatoid liver carcinoma composed of atypical spindle cells without features of either HCC or ICC: a case report. Int J Clin Exp Med. 2016;9(10):20308-20313.

6. Haratake J, Horie A. An immunohistochemical study of sarcomatoid liver carcinomas. Cancer. 1991;68(1):93-97.

7. Gu Q, Yu X, Chen H, Chen G. Clinicopathological features of combined hepatocellularcholangiocarcinoma with sarcomatous change. Medicine. 2018;97(3).

8. Ines DD, Bailly A, Lannareix V, et al. Hepatocellular carcinoma with sarcomatous change: prompt and fatal intraabdominal recurrence after liver transplantation. Gastroentérologie Clinique et Biologique. 2009;33(6-7):590-593.

9. Lin $M$, Huang $H$. Recurrent Hepatic Sarcomatoid carcinoma cured by doxorubicin and ifosfamide chemotherapy. 2015; Sep. Journal of Cancer 
Research and Practice. https://doi.org/10.6323/

JCRP.2015.2.3.07

10. Liao S-H, Su T-H, Jeng Y-M, et al. Clinical manifestations and outcomes of patients with sarcomatoid hepatocellular carcinoma. Hepatology. 2019;69(1):209-221.

\section{ACKNOWLEDGEMENTS}

We acknowledge Niru Nahar, MBBS (Assistant Professor in the Department of Pathology, Joan C. Edwards School of Medicine) for her assistance. 